\title{
Worst-Case Minimum Rectilinear Steiner Trees in All Dimensions
}

\author{
Timothy Law Snyder \\ Department of Computer Science, Georgetown University, \\ Washington, DC 20057, USA \\ tim@normal.georgetown.edu
}

\begin{abstract}
It is proved that the length of the longest possible minimum rectilinear Steiner tree of $n$ points in the unit $d$-cube is asymptotic to $\beta_{d} n^{(d-1) / d}$, where $\beta_{d}$ is a constant that depends on the dimension $d \geq 2$.

A method of Chung and Graham (1981) is generalized to dimension $d$ to show that $1 \leq \beta_{d} \leq d 4^{(1-d) / d}$. In addition to replicating Chung and Graham's exact determination of $\beta_{2}=1$, this generalization yields new bounds such as $1 \leq \beta_{3}<1.191$ and $1 \leq \beta_{4} \leq \sqrt{2}$.
\end{abstract}

\section{Introduction}

The purpose of this paper is to investigate the worst-case length of a minimum Steiner tree of $n$ points in bounded $d$-space under the rectilinear $\left(L_{1}\right)$ metric. For this purpose, we restrict our attention to sets of $n$ points in the unit $d$-cube, $[0,1]^{d}$.

Let $S$ be a set of $n$ points in $[0,1]^{d}$. A Steiner tree of $S$ is a set of edges or wires incident with a point set $S^{\prime}$ where $S \subseteq S^{\prime}$. The points in $S^{\prime}-S$, i.e., those in the graph of the Steiner tree but not in the original point set $S$, are called Steiner points. In the construction of minimum Steiner trees, we associate a cost or distance function with each wire. Let $x=\left(x_{1}, x_{2}, \ldots, x_{d}\right)$ and $y=\left(y_{1}, y_{2}, \ldots, y_{d}\right)$ be points belonging to $[0,1]^{d}$. For the wire $w=\{x, y\}$, let $d_{\mathrm{R}}(w)$ be the rectilinear distance $\left|x_{1}-y_{1}\right|+\left|x_{2}-y_{2}\right|+\cdots+\left|x_{d}-y_{d}\right|$. Rectilinear distance is often referred to as the Manhattan or $L_{1}$ metric.

A rectilinear Steiner tree is one in which the length of the wire $w$ is taken to be $d_{\mathrm{R}}(w)$. In general, we can geometrically represent a wire of a rectilinear Steiner tree in the $d$-cube by connecting its endpoints with contiguous orthogonal line segments that parallel a subset of the $d$ coordinate axes.

A minimum rectilinear Steiner tree (MRST) of the point set $S$ is a Steiner tree of length $\min _{T}\left\{\sum_{\omega \in T} d_{R}(w): T\right.$ is a rectilinear Steiner tree of $\left.S\right\}$. Our object of 
primary interest will be the sequence

$$
s^{*}(n)=\max _{\substack{s \in[0.1]^{d} \\|S|=n}} \min _{T}\left\{\sum_{w \in T} d_{\mathrm{R}}(w): T \text { is a rectilinear Steiner tree of } S\right\}
$$

Since the minimized quantity in (1.1) is an MRST of the point set $S$, we call $s^{*}(n)$ the worst-case length of a minimum rectilinear Steiner tree of $n$ points in the unit $d$-cube.

Two things should be noted concerning the definition of $s^{*}(n)$. First, for brevity, we have masked the dependence of $s^{*}(n)$ on the dimension $d$. Since we will at all times consider $d$ to be fixed, we will find it convenient to follow this convention in our notation. Second, we use max in place of sup in (1.1) because the supremum is attainable (Chung and Graham (1981)).

In the sections that follow, we prove two main results. Theorem 1 tells us that the quantity $s^{*}(n) / n^{(d-1) / d}$ converges to a constant in dimensions two and greater:

Theorem 1. There exists a positive constant $\beta_{d}$ depending on the dimension $d \geq 2$ such that as $n \rightarrow \infty$,

$$
s^{*}(n) \sim \beta_{d} n^{(d-1) / d} .
$$

Theorem 2 gives us upper and lower bounds for the rectilinear constant $\beta_{d}$ :

Theorem 2. For all $d \geq 1$,

$$
1 \leq \beta_{d} \leq d 4^{(1-d) / d}
$$

Though it is often assumed that the worst-case length of an MRST of $n$ points is exactly asymptotic to a constant times $n^{(d-1) / d}$, Theorem 1 is the first result to this effect for general $d$. Chung and Graham (1981) showed that, in dimension two, $s^{*}(n) \leq \sqrt{n}+1+o(1)$, which, when combined with a lower bound of $s^{*}(n) \geq$ $\sqrt{n}+O(1)$ obtained by a regular square lattice packing of the cube, yields the exact determination $\beta_{2}=1$. For $d \geq 3$, however, there exist no previous results strong enough to guarantee the existence of the $\beta_{d}$ as does Theorem 1 .

Theorem 2 reproduces Chung and Graham's value $\beta_{2}=1$ and gives the first upper bounds on the values of $\beta_{d}$ for $d \geq 3$, but leaves the problem of exact values in these dimensions open. The lower bound in Theorem 2 is from Snyder (1991).

Since the values of the constants $\beta_{d}$ have been relatively elusive, attempts have been made to relate them to more easily obtained constants. A classical bound of Hwang (1976) is that, for any point set $V \subset \mathbb{R}^{2}$, if we let MRST( $V$ ) and MST( $V$ ) be the rectilinear lengths of minimum Steiner and minimum spanning trees of $V$, respectively, then $\operatorname{MST}(V) \leq \frac{3}{2} \operatorname{MRST}(V)$. Also in $d=2$, Chung and Hwang (1979) let $\operatorname{RECT}(V)$ be the length of the semiperimeter of the smallest rectangle with vertical and horizontal lines that encloses $V$, and proved that if $|V|=n$, then

$$
\max _{V \in[0,1]^{2}} \frac{\operatorname{MRST}(V)}{\operatorname{RECT}(V)} \sim \frac{\sqrt{n}}{2}
$$


as $n \rightarrow \infty$. They also gave exact values for the ratio in (1.4) when $6 \leq n \leq 10$, complementing those of Hanan (1966) for smaller $n$. Prior to this result, it was believed that the length of the semiperimeter of the smallest enclosing rectangle for a given set of points was a reasonable estimate of the length of an MRST of the points.

Much of what is known about the exact length of the MRST is in probabilistic settings. In particular, Bern (1988) gave a probabilistic result that separates the constants associated with the rectilinear versions of the Steiner tree and minimum spanning tree problems in the unit square. Specifically, if $C_{\text {MST }} \sqrt{N}$ and $C_{\text {Mrst }} \sqrt{N}$ are the expected lengths of rectilinear minimum spanning and Steiner trees, respectively, of points distributed according to a Poisson process with intensity $N$ in $\mathbb{R}^{2}$, then $C_{\text {MRST }} \leq C_{\text {MST }}-0.0014$ as $N \rightarrow \infty$. Bern also mentioned that it is simple to show that both of these constants are at least $\sqrt{2 \pi} / 8$.

Also under the umbrella of MRSTs in probabilistic settings is the following asymptotic result. The theorem of Beardwood et al. (1959) or Steele's theory of subadditive Euclidean functionals (Steele (1981)) can be used to show that, if $R_{n}$ is the length of an MRST of $X_{1}, X_{2}, \ldots, X_{n}$, where the $X_{i}$ are uniformly distributed on $[0,1]^{d}$, then with probability one,

$$
R_{n} \sim \gamma_{d} n^{(d-1) / d}
$$

where $\gamma_{d}$ is a constant depending only on the dimension $d \geq 2$.

The MRST problem has applications that include optimal arrangements of wiring in VLSI circuits, optimal communication networks, and the determination of evolutionary ancestors of known species in the life sciences (see, e.g., Gilbert and Pollak (1968) and Foulds et al. (1979)). Unlike the minimum spanning tree problem, which forms a minimum-length tree on a given set of points without the ability to augment the point set with additional points, the MRST problem is $N P$-complete for a discretized version of the $L_{1}$ metric (see Garey et al. (1976) and Garey and Johnson (1977)). Hanan (1966) proved the existence of a finite method for the MRST problem in the plane. In general dimension, Sankoff and Rousseau (1975) designed an algorithm for the MRST, and Snyder (1992) did the same by generalizing Hanan's algorithm to dimensions $d>2$. The rectilinear algorithm of Snyder (1992) runs faster than that of Sankoff and Rousseau (1975), but the Sankoff and Rousseau algorithm is valid for any metric.

It is no longer surprising that the probabilistic rate of growth expressed by (1.5) is so similar in form to the growth rate of a worst-case MRST expressed by Theorem 1. For example, Steele and Snyder (1989) and Snyder and Steele (1990) proved similar worst-case rates of growth for the Euclidean traveling salesman, minimum spanning tree, and greedy matching problems, as did Snyder (1987) for the Euclidean minimum matching problem. All of these problems, however, have edge weights determined by the Euclidean metric as opposed to the rectilinear metric used here.

The Steiner problem also has the added intricacy of possible augmentation of the point set on which the tree is formed, and, since there is no known polynomial algorithm for the Steiner problem, our analysis is devoid of algorithmic characterizations and constructions of a Steiner tree. Hence, new methods are required to 
overcome these difficulties specific to the Steiner problem. Particularly necessary is the generalization of Hanan's theorem used in Section 2.

This paper can be roughly divided into two independent parts: Sections 5 through 7 establish the bounds on the $\beta_{d}$ of Theorem 2, and Sections 2 through 4 prove Theorem 1 , which establishes the existence of the $\beta_{d}$. In the next section, Section 2, we present two recursive inequalities that tell us exactly what will be needed to prove Theorem 1, and we commence with the proof of Theorem 1 by recursively constructing a nearly worst-case rectilinear Steiner tree. Section 3 then assembles some geometric facts that make easy the proof of Theorem 1 in Section 4.

\section{A Nearly Worst-Case Minimum Rectilinear Steiner Tree}

We will prove Theorem 1 by using the following general lemma, which makes explicit two relationships that will be sufficient to prove that $s^{*}(n) \sim \beta_{d} n^{(d-1) / d}$ as $n \rightarrow \infty$. As we shall see shortly, despite their technical appearance, the relationships (2.1(i)) and (2.1(ii)) of the lemma are naturally related to geometric quantities such as $s^{*}(n)$. The inequality (2.1(i)) will be used to tell us that $s^{*}(n)$ has a slow incremental rate of growth, while $(2.1(\mathrm{ii}))$ will indicate an approximate recursiveness in the length of a worst-case MRST.

Lemma 2.1. If $\rho(1)=0$ and there exists some $c \geq 0$ such that for all $m \geq 1$ and $k \geq 1$

$$
\begin{aligned}
& \text { (i) } \rho(n+1) \leq \rho(n)+c n^{-1 / d} \text { and } \\
& \text { (ii) } m^{d-1} \rho(k)-m^{d-1} k^{(d-1) / d} r(k) \leq \rho\left(m^{d} k\right),
\end{aligned}
$$

where $r(k) \rightarrow 0$ as $k \rightarrow \infty$, then as $n \rightarrow \infty$,

$$
\rho(n) \sim \beta n^{(d-1) / d}
$$

for a positive constant $\beta$.

This lemma has been instrumental in proving worst-case asymptotic results similar to Theorem 1 for other quantities of combinatorial optimization (Snyder (1987), Steele and Snyder (1989), and Snyder and Steele (1990)). Though we do not prove the lemma here, one can consult these references for proofs.

We begin our proof of Theorem 1 by recursively constructing an MRST that is of nearly worst-case length. We will then use this to develop a recursion for $s^{*}(n)$ that satisfies Condition (ii) of Lemma 2.1. Condition (i) will follow almost immediately from the analysis that deals with Condition (ii).

We first divide the $d$-cube into $m^{d}$ equally-sized smaller cubes of side length $1 / m$, where $m \geq 1$. The boundaries of these cubes form a grating, which we fatten to a width $\delta$, where $0<\delta<1 / m$ will be explicitly chosen later. This leaves us with $m^{d}$ cells, each of which is a cube having side length $1 / m-\delta$, with each centered inside one of the cubes of side length $1 / m$. Label the cells $Q_{1}, Q_{2}, \ldots, Q_{m^{d}}$ and consider $V$, an arrangement of $k$ points that attains the worst-case length $s^{*}(k)$ 
when placed inside $[0,1]^{d}$. By scaling this arrangement to the cube $[0,1 / m-\delta]^{d}$ and by translation, we can place a rescaled copy of $V$ in each of the $m^{d}$ cells. We use $V_{i}$ to denote the set of $k$ points placed in the cell $Q_{i}$, where $1 \leq i \leq m^{d}$.

Consider an MRST of the $m^{d} k$ points of $\bigcup_{i=1}^{m^{d}} V_{i}$. If we pass, through each point of $\bigcup_{i=1}^{m^{d}} V_{i}, d$ hyperplanes normal to the coordinate axes, then we obtain a grid (hypergrid) in the $d$-cube. Let $H\left(\bigcup_{i=1}^{m^{d}} V_{i}\right)$ be this grid. For every set of $d$ mutually orthogonal hyperplanes in $H\left(\bigcup_{i=1}^{m^{d}} V_{i}\right)$ there is a point where the hyperplanes intersect. The following theorem is adapted from Snyder (1992), which contains a $d$-dimensional analog to a theorem of Hanan (1966).

Theorem There exists an MRST $T$ of $\bigcup_{i=1}^{m^{d}} V_{i} \subset[0,1]^{d}$ such that if $v$ is a Steiner point of $T$, then $v$ coincides with an intersection point of $H\left(\bigcup_{i=1}^{m^{t}} V_{i}\right)$.

This theorem tells us that we can select an MRST $T$ of $\bigcup_{i=1}^{m^{d}} V_{i}$ such that if $v$ is a Steiner point of the graph of $T$, then $v \in \bigcup_{i=1}^{m^{d}} Q_{i}$, i.e., each Steiner point $T$ is in some cell $Q_{i}$ and not in the space of width $\delta$ that separates the cells. This feature of $T$ is critical in what follows.

For all $1 \leq i \leq m^{d}$, let $T_{i}$ be an MRST of $V_{i}$. To form a recursion like Condition (ii) of Lemma 2.1, we will compare the length of $T$ with the combined length of $\bigcup_{i=1}^{m^{d}} T_{i}$. To do this, we first remove from $T$ all the wires $w$ such that $d_{\mathrm{R}}(w)>\delta$. Since $T$ has no Steiner points in the space separating the cells, any wires in $T$ that pass through the intercellular space must have rectilinear length at least $\delta$, hence all these wires are removed. What remains is a forest of rectilinear Steiner trees, with each tree entirely contained inside some cell $Q_{i}$. Some of the $Q_{i}$ may contain more than one tree or no nontrivial trees at all.

After removing the relatively long wires of $T$, within each cell we connect the trees in the cell by inserting wires until we obtain a single tree that spans all the cell's points. This gives us a forest of $m^{d}$ rectilinear Steiner trees with each cell containing exactly one tree. These trees will most likely be suboptimal.

It is simple to estimate the combined length of this forest. Let $\lambda(\delta)$ be the number of wires that were removed from $T$, i.e., let

$$
\lambda(\delta)=|\{w \in T:|w|>\delta\}|
$$

We began with a MRST of length $\sum_{w \in T} d_{R}(w)$, from which we removed $\lambda(\delta)$ wires to obtain a maximum of $\lambda(\delta)+1$ connected components, each contained entirely within a cell. We then added wires inside the cells, so each wire added has a maximum length of $d(1 / m-\delta)$, where we have scaled $d$, the maximum rectilinear wire length in the $d$-cube, to reflect the size of a cell. To connect all the within-cell components requires at most $\lambda(\delta)$ wires, so the total cost of the wires added is at most $d \lambda(\delta)(1 / m-\delta)$. So, what remains is bounded from above by

$$
\sum_{w \in T} d_{\mathrm{R}}(w)+d \lambda(\delta)\left(\frac{1}{m}-\delta\right)
$$

But, since each cell contains a rectilinear Steiner tree, the length of a cell's tree is at least the length of the MRST $T_{i}$ formed on its $k$ points, which, by definition, 
is $s^{*}(k)(1 / m-\delta)$, so

$$
m^{d}\left(\frac{1}{m}-\delta\right) s^{*}(k) \leq \sum_{w \in T} d_{\mathrm{R}}(w)+d \lambda(\delta)\left(\frac{1}{m}-\delta\right) .
$$

From this, since $\sum_{w \in T} d_{\mathrm{R}}(w) \leq s^{*}\left(m^{d} k\right)$, we obtain

$$
m^{d-1} s^{*}(k)-\left[\delta m^{d} s^{*}(k)+\frac{d}{m} \lambda(\delta)\right] \leq s^{*}\left(m^{d} k\right)
$$

which is not unlike Condition (2.1(ii)) of Lemma 2.1.

In the next section, we present some geometric lemmas that form an interesting bound on the quantity $\lambda(\delta)$, with an added bonus of demonstrating Condition (2.1(i)) for $s^{*}(n)$.

\section{Incremental Rates of Growth and a Bound on the Number of Long Wires that Is Independent of $n$}

In this section we present a series of lemmas that includes a bound in the incremental rate of growth of the sequence $s^{*}$, as well as a bound on the number of wires of length $\delta$ or greater in an MRST of $n$ points in the cube. A main virtue of our bound on the number of long wires is that it is independent of the number of points. This will assist us in estimating $\lambda(\delta)$ when we return to the recursion (2.5) of the previous section.

We begin with a simple but fundamental lemma that results from the pigeonhole principle.

Lemma 3.1. If $V \subset[0,1]^{d}$ is a set of $n$ points, then there exists a constant $c_{1}$ such that

$$
\max _{V} \min _{u, v \in V} d_{\mathbf{R}}(\{u, v\}) \leq c_{1} n^{-1 / d}
$$

Proof. Cover each point $v$ of $V$ with a $d$-cube of side length $t$ centered at $v$. The resulting $n$ cubes must be entirely contained in the cube $[-t / 2,1+t / 2]^{d}$, which has volume $(1+t)^{d}$. If we assume that the intersection of the $n$ smaller cubes is empty, then they comprise a combined volume of $n t^{d}$, hence, $n t^{d} \leq(1+t)^{d} \leq 2^{d}$ and $t \leq 2 n^{-1 / d}$. Allowing $t$ to be as large as possible until a pair of cubes centered at some $u$ and $v$ meet, it is clear that $d_{\mathrm{R}}(\{u, v\}) \leq d t$, giving a bound of $c_{1} \leq 2 d$.

Lemma 3.1 literally sets the scale for the rectilinear lengths of objects such as MRSTs in the cube. We make no attempt to obtain a tight bound on $c_{1}$, for we will be interested only in rates of growth in what follows.

The next lemma guarantees the existence of a certain relatively short wire in any MRST, namely, a short wire whose endpoints are not both Steiner points. 
Lemma 3.2. Let $T$ be an MRST of $V \subset[0,1]^{d}$ where $|V|=n$. Then there exists a constant $c_{2}$ and $a$ wire $w=\{u, v\} \in T$ such that

$$
\text { (i) } d_{\mathrm{R}}(w) \leq c_{2} n^{-1 / d}
$$

and

$$
\text { (ii) } u \in V \text { or } v \in V \text {. }
$$

Proof. To prove both (i) and (ii), we note that by Lemma 3.1 there exists a pair of points $x \in V$ and $y \in V$ such that $d_{\mathbf{R}}(\{x, y\}) \leq c_{1} n^{-1 / d}$. Suppose that $T$, the MRST of $V$, does not contain the wire $\{x, y\}$, and let $\left\{x^{\prime}, x\right\}$ and $\left\{y^{\prime}, y\right\}$ be wires of $T$ incident with $x$ and $y$.

Since $\{x, y\}$ is not in $T$, then adding it to $T$ creates a cycle $C$ in the graph of $T$. Moreover, if $\left\{x^{\prime}, x\right\} \notin C$ or $\left\{y^{\prime}, y\right\} \notin C$, then there must exist two wires of $T$ incident with $x$ and $y$ that do belong to the cycle, so we can assume without loss that $\left\{x^{\prime}, x\right\} \in C$ and $\left\{y^{\prime}, y\right\} \in C$. Now, by the minimality of $T$, both $d_{\mathrm{R}}\left(\left\{x^{\prime}, x\right\}\right) \leq$ $d_{\mathrm{R}}(\{x, y\})$ and $d_{\mathrm{R}}\left(\left\{y^{\prime}, y\right\}\right) \leq d_{\mathrm{R}}(\{x, y\}) \leq c_{1} n^{-1 / d}$, proving (i) with $c_{2}=c_{1}$. In addition, since $x \in V$ and $y \in V$, (ii) is also satisfied.

The existence of such a relatively short wire in any MRST leads us to the following lemma, which guarantees a slow incremental rate of growth for $s^{*}$.

Lemma 3.3. There exists a constant $c_{3}$ such that, for all $n \geq 1$,

$$
s^{*}(n+1) \leq s^{*}(n)+c_{3} n^{-1 / d} .
$$

Proof. Let $V$ be a set of $n+1$ points in $[0,1]^{d}$ having an MRST of total length $s^{*}(n+1)$. By Lemma 3.2(i) an MRST of $V$ must contain a wire $\{u, v\}$ satisfying $d_{\mathrm{R}}(\{u, v\}) \leq c_{2}(n+1)^{-1 / d} \leq c_{2} n^{-1 / d}$. By Lemma 3.2(ii) at least one of the points $u$ and $v$ must belong to $V$, so, without loss, we assume that $u \in V$. Remove $u$ from $V$ and let $T$ be an MRST of $V-\{u\}$. If we now add the wire $\{u, v\}$ to $T$ we obtain a rectilinear Steiner tree of $V$ whose length majorizes the minimal length $s^{*}(n+1)$. This yields

$$
s^{*}(n+1) \leq \sum_{w \in T} d_{\mathrm{R}}(w)+d_{\mathrm{R}}(\{u, v\}) \leq s^{*}(n)+c_{2} n^{-1 / d},
$$

which is (3.4) with $c_{3}=c_{2}$.

The following lemma gives us a general inequality that bounds the total length of an arbitrary subset of wires of any MRST.

Lemma 3.4. If $W$ is a subset of wires of an MRST of $n$ points in $[0,1]^{d}$, then there exists a constant $c_{4}$ such that

$$
\sum_{w \in W} d_{\mathrm{R}}(w) \leq c_{4}|W|^{(d-1) / d} .
$$


Proof. First, we borrow a lemma from Steele and Snyder (1989), where the analog to inequality (3.6) is proved for Euclidean minimum spanning trees. Using Lemma 3.1 , it is easy to show that the Euclidean inequality of Steele and Snyder (1989) also holds for rectilinear minimum spanning trees, up to a constant factor. To summarize this result, if we let $E$ be a subset of edges of a rectilinear minimum spanning tree of a set of points in $[0,1]^{d}$, then there exists a constant $c$ such that

$$
\sum_{e \in E} d_{\mathrm{R}}(e) \leq c|E|^{(d-1) / d} .
$$

Let $V$ be a set of points of $[0,1]^{d}$ and let $S$ be an MRST of $V$. It is trivial to note that if $V^{\prime}$ is the set of Steiner points added to $V$ to form $S$, and if $T$ is a rectilinear minimum spanning tree of $V \cup V^{\prime}$, then $\sum_{w \in S} d_{\mathbf{R}}(w)=\sum_{e \in T} d_{\mathbf{R}}(e)$. In other words, $S$ is a minimum spanning tree of $V \cup V^{\prime}$. Since this is true, we can immediately replace the set $E$ with the set $W$ in inequality (3.7), proving the lemma.

We now have assembled the tools necessary for our bound on the number of long wires in an MRST in the $d$-cube. Define $v(x, n)$ to be the maximum number of wires $w$ of an MRST of $n$ points in $[0,1]^{d}$ such that $d_{\mathrm{R}}(w) \geq x$. Formally, let

$$
v(x, n)=\max _{\substack{V \subset[1,1]^{4} \\|V|=n}} \mid\left\{d_{\mathbf{R}}(w) \geq x: w \in T \text { and } T \text { is an MRST of } V\right\} \mid
$$

Intriguingly, Lemma 3.5 gives us a bound on $v(x, n)$ that is independent of the number of points.

Lemma 3.5. For all $n \geq 1$ and $x>0$, there exists a constant $c_{5}$ such that

$$
v(x, n) \leq \frac{c_{5}}{x^{d}}
$$

Proof. Let $T$ be an MRST of a set of $n$ points in $[0,1]^{d}$ and let

$$
\psi_{T}(x)=\left|\left\{w \in T: d_{\mathrm{R}}(w) \geq x\right\}\right|
$$

By Lemma 3.4,

$$
x \psi_{T}(x) \leq \sum_{\substack{w \in T \\ d_{R}(w) \geq x}} d_{\mathrm{R}}(w) \leq c_{4}\left\{\psi_{T}(x)\right\}^{(d-1) / d} ;
$$

solving (3.11) for $\psi_{T}(x)$ yields $\psi_{T}(x) \leq\left(c_{4} / x\right)^{d}$. Since this relationship holds for an MRST $T$ of any $n$-set, it holds for a tree having the maximum number of wires of length $x$ or greater, which proves the lemma with $c_{5}=c_{4}^{d}$.

The lemmas of this section simplify the proof of Theorem 1, which is the task of the next section. 


\section{The Worst-Case Length of an MRST}

In this section we use the recursive construction from Section 2 along with Lemma 2.1 and the bounds of Section 3 to prove Theorem 1, which gives us the exact asymptotic length of a worst-case MRST of $n$ points.

Our objective is to show that conditions (2.1(i)) and (2.1(ii)) of Lemma 2.1 are true when we replace the function $\rho$ of the lemma with the function $s^{*}$. We begin by recalling inequality $(2.5)$. Using Lemma 3.5 , we can bound $\lambda(\delta)$ in $(2.5)$ to obtain

$$
m^{d-1} s^{*}(k)-\left[\delta m^{d} s^{*}(k)+\frac{c_{5} d}{m \delta^{d}}\right] \leq s^{*}\left(m^{d} k\right) .
$$

To bound the quantity $s^{*}(k)$ we will need one more lemma.

Lemma 4.1. For all $n \geq 1$, there exists a constant $c_{6}$ such that

$$
s^{*}(n) \leq c_{6} n^{(d-1) / d} .
$$

Proof. One way to obtain (4.2) is by replacing $n$ with $i$ in inequality (3.4) and summing both sides from $i=1$ to $i=n-1$.

An alternative proof uses a classical bound mentioned in Courant and Robbins (1941) and formally proved in Melzak (1961) (and reviewed in Hanan (1966) and Gilbert and Pollak (1968)), which says that if we let $k$ be the number of Steiner points in an MRST of $n$ points, where $n \geq 2$, then $0 \leq k \leq n-2$. Though this bound was originally proved for dimension two, it can be easily generalized to all dimensions. Since a tree constructed on $n$ points has $n-1$ edges, the bounds on the number of Steiner points tell us that an MRST of $n \geq 2$ points has $n-1 \leq n+k-1 \leq 2 n-3$ wires. From Lemma 3.4 we obtain

$$
s^{*}(n) \leq c_{5}(2 n-3)^{(d-1) / d}
$$

which proves (4.2) with $c_{6}=2^{(d-1) / d} c_{5}$.

Applying Lemma 4.1 to inequality (4.1) gives

$$
m^{d-1} s^{*}(k)-\left[c_{6} \delta m^{d} k^{(d-1) / d}+\frac{c_{5} d}{m \delta^{d}}\right] \leq s^{*}\left(m^{d} k\right)
$$

and, if we approximately minimize the bracketed expression in (4.4) by choosing

$$
\delta=m^{-1} k^{(1-d) / d(d+1)}
$$

then we see that there exists a constant $c_{7}$ such that

$$
m^{d-1} s^{*}(k)-c_{7} m^{d-1} k^{(d-1) /(d+1)} \leq s^{*}\left(m^{d} k\right) .
$$

This recursion is precisely Condition (2.1(ii)) of Lemma 2.1 for the function $s^{*}$, with $r(k)=c_{7} k^{(1-d) / d(d+1)}$. 
Condition (2.1(i)) of Lemma 2.1 has already been proved by Lemma 3.3, hence we have satisfied for $s^{*}$ all the conditions required by Lemma 2.1. This proves Theorem 1.

Theorem 1 proves the existence of the constants $\beta_{d}$; in the next three sections we turn our attention to the values of these constants, with the goal of bounding them for all $d$, to obtain Theorem 2 .

\section{Chung and Graham Strips in Dimension Three}

The next three sections establish the bounds on the $\beta_{d}$ presented in Theorem 2 . In this section we begin our investigation of bounds on the $\beta_{d}$ by extending a method of Chung and Graham (1981) to $d=3$. The general case of dimension $d$ is then handled in Section 6, wherein we simultaneously construct $2^{d}$ heuristic rectilinear Steiner trees. The analysis is completed in Section 7, which determines the combined length of the set of heuristic trees.

Before treating upper bounds we first note from Snyder (1991) that

$$
\beta_{d} \geq 1 \text { for all } d \geq 1 \text {. }
$$

This provides the lower bound of Theorem 2 .

From this point, we focus our attention on upper bounds. The traditional method for obtaining these bounds is by constructing heuristic rectilinear Steiner trees based on a set of "strips" that span the cube, in the spirit of Few (1955). Chung and Graham (1981) closed the book on $\beta_{2}$ using such a heuristic to prove that $\beta_{2}=1$, and our bounds on $\beta_{d}$ are based on a generalization of their method.

We first consider $[0,1]^{3}$, in which we will simultaneously construct four heuristic rectilinear Steiner trees. To carry out the construction label the coordinate axes $x, y$, and $z$. Along the $z$-axis, from $z=0$ to $z=1$, establish $m+1$ $z$-levels: $z_{0}=0, z_{1}=1 / m, z_{2}=2 / m, \ldots, z_{m}=1$, where $m \geq 1$. In the plane at each $z$-level $z_{i}$, where $0 \leq i \leq m$, divide the unit square into $m$ regions (strips) by placing $m+1$ line segments parallel to the $x$-axis and at a distance of $1 / m$ from each other, each having its endpoints in the planes $x=0$ and $x=1$. Of course, at each level, two of these segments constitute half of the boundary of the square at level $z_{i}$, namely, the intersection of the boundary with the planes $y=0$ and $y=1$. We include the remainder of the boundary of the square, which is in the planes $x=0$ and $x=1$, as well. Finally, we connect the $m+1 z$-levels by four line segments that parallel the $z$-axis, with each one connecting the corners of the unit squares at $z=0$ and $z=1$.

We call the resulting basic framework of strips a Chung and Graham 3-Strip.The portion of the 3-Strip at each $z$-level is identical to the construction used by Chung and Graham (1981) in $d=2$; for this reason, we call it a Chung and Graham 2-Strip. So, a Chung and Graham 3-Strip is simply a set of $m+1$ Chung and Graham 2-Strips, each occupying a distinct $z$-level, which are connected together by the four unit segments that parallel the $z$-axis. Figure 1 depicts a Chung and Graham 3-Strip with $m=3$; the different types of segments in the figure will be explained shortly. 


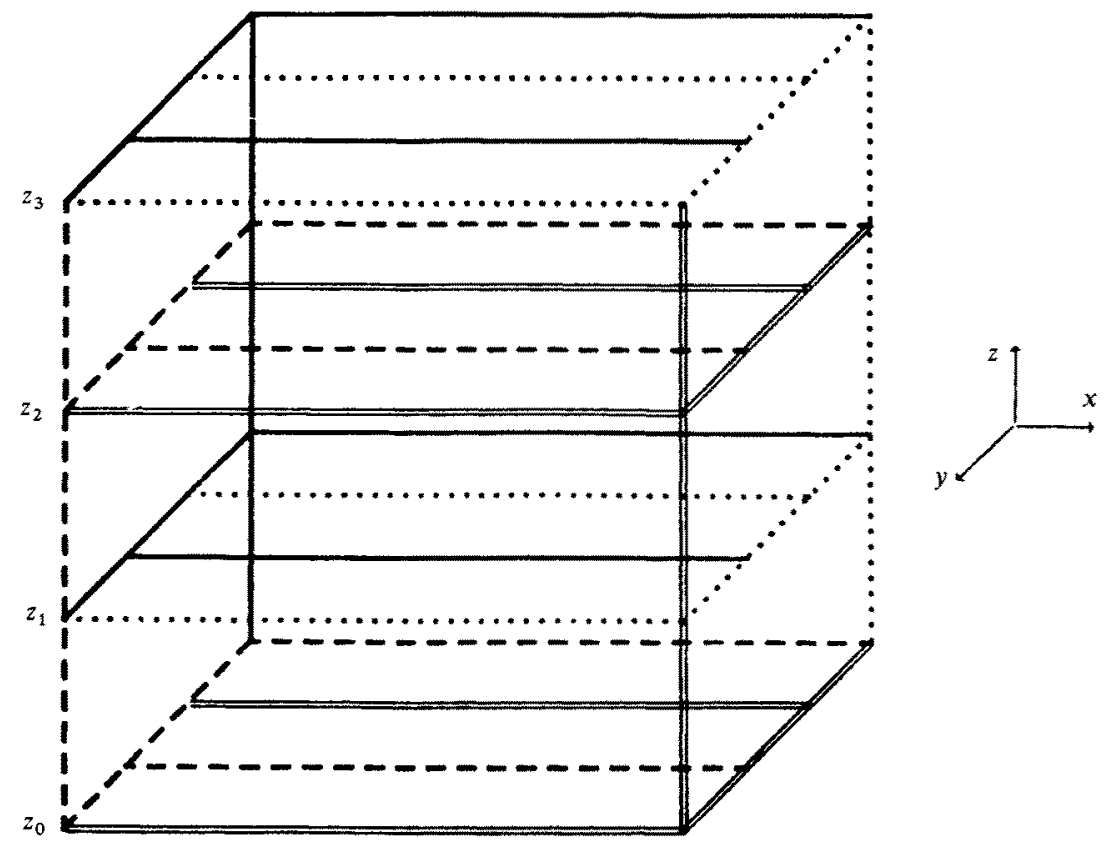

Fig. 1. The Chung and Graham 3-Strip and its assignment to four trees. Here, $m=3$.

Our set of four rectilinear Steiner trees of a given set of $n$ points will consist of the Chung and Graham 3-Strip and a set of relatively short wire segments called juttings, which serve to connect the points to the 3-Strip, providing they do not already lie directly on it.

Each line segment of the 3-Strip will belong to exactly one of the four Steiner trees. To determine the tree to which each segment of the 3-Strip belongs, we designate $z$-level $z_{i}$ to be an even $z$-level if $i$ is even, and an odd $z$-level if $i$ is odd, where $0 \leq i \leq m$. Of the four trees, two will contain no line segments from the odd $z$-levels, and the other two will contain no line segments from even $z$-levels. We call these pairs of trees even trees and odd trees, respectively.

We will refer to the trees under construction as $T_{e 0}, T_{e 1}, T_{o 0}$, and $T_{01}$, with the trees $T_{e i}$ being even and the trees $T_{o i}$ being odd, where $i=0,1$. At each even $z$-level we alternately assign each of the $m+1$ segments parallel to the $x$-axis to the trees $T_{e 0}$ and $T_{e 1}$, respectively. Similarly, we alternately assign the segments paralleling the $x$-axis at odd $z$-levels to the odd trees. These segments at each $z$-level are then connected by the two segments of the level that parallels the $y$-axis, with the segments in the plane $x=0$ belonging to $T_{e 0}$ and $T_{o 0}$ and those of the plane $x=1$ belonging to $T_{e 1}$ and $T_{01}$. The only segments of the 3-Strip that remain are the four segments paralleling the $z$-axis. We use these segments to connect the $z$-levels, this is done by arbitrarily assigning each segment to a distinct tree.

We have now assigned each segment of the Chung and Graham 3-Strip to one of the four trees. Figure 1 depicts a Chung and Graham 3-Strip with $m=3$, along 


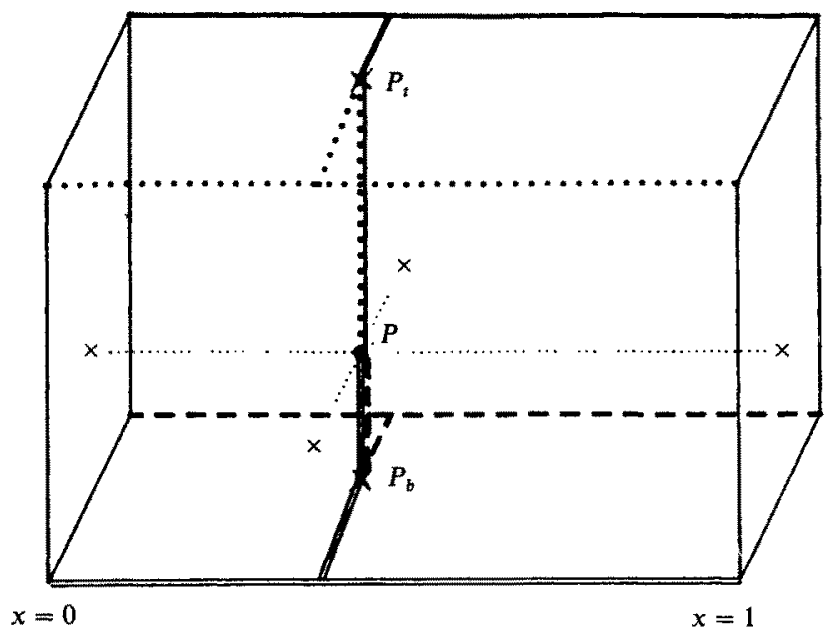

Fig. 2. A 3-box and a set of four juttings connecting point $P$ via $P_{\mathrm{t}}$ and $P_{\mathrm{b}}$ to the 3-Strip.

with the assignments, where the even trees are represented, respectively, by dashed and doubled line segments, and the odd trees by dotted and solid segments.

If we let $J$ be the set of juttings that connect the set of $n$ points to the 3-Strip, then it is easily seen that the total length of the four trees using the Chung and Graham 3-Strip is

$$
T_{e 0}+T_{e 1}+T_{o 0}+T_{o 1}=(m+1)^{2}+4+\sum_{w \in J} d_{R}(w) .
$$

All that remains is to reach the points by juttings and assess the cost entailed in doing so.

Since we have four trees, we will need four juttings for each point. Figure 2 depicts a typical set of juttings. In particular, the picture shows the upper front rectangular section, i.e., the intersection of the top third with the front third, of the cube in Figure 1, from $x=0$ (at the left) to $x=1$ (at the right). Inside the rectangular solid in Figure 2 is a point $P$ that has to be connected via juttings to the 3-Strip, which is represented here by the four edges of the solid that parallel the $x$-axis (traveling from left to right in the figure). Let $P_{\mathrm{t}}$ and $P_{\mathrm{b}}$ be projections of $P$ onto the top and bottom planes (adjacent $z$-levels), respectively. We first connect both trees in the 3-Strip at the top $z$-level to $P_{t}$ and both trees at the bottom $z$-level to $P_{\mathrm{b}}$ with juttings that parallel the $y$-axis, as shown in the figure. Both trees of the top level then reach $P$ from $P_{t}$ by a jutting parallel to the $z$-axis, and the trees of the bottom level reach $P$ in similar fashion from $P_{b}$.

It is easily seen that the scenario depicted in Figure 2 is analogous to what we would see were we to select any other of the nine rectangular sections of the cube depicted in Figure 3. Each section contains four distinct unit segments of the 3-Strip that belong to $T_{e 0}, T_{e 1}, T_{o 0}$, and $T_{o 1}$, so we can reach all of the $n$ points using juttings as we have in Figure 2. 


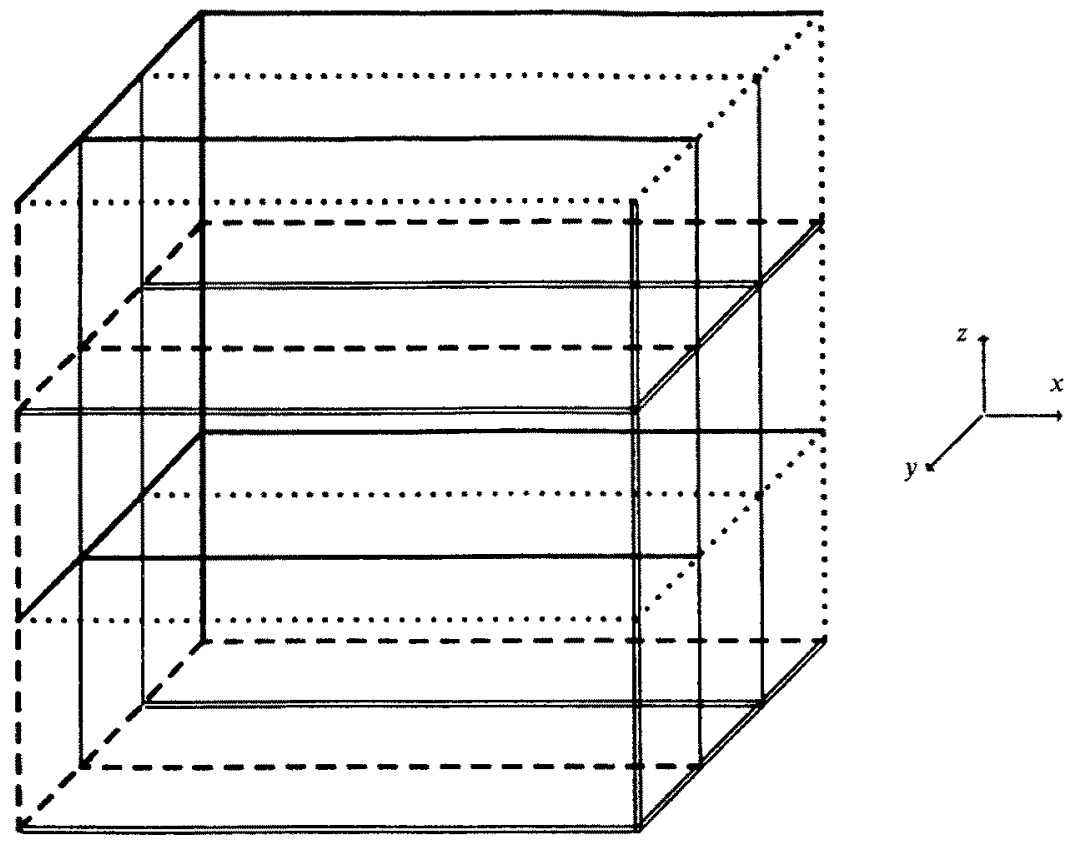

Fig. 3. The nine $d$-boxes in dimension three when $m=3$.

The total cost of the juttings from the top-level trees to $P_{t}$ is $1 / m$, as is the total cost of the bottom-level trees to $P_{\mathrm{b}}$. The juttings from $P_{\mathrm{t}}$ and $P_{\mathrm{b}}$ to $P \operatorname{cost} 2 / \mathrm{m}$, for each of these juttings is traversed by two trees. Hence. the total jutting cost for a point that does not lie directly on the 3-Strip is $4 / m$. Using (5.2), this gives us a combined cost of

$$
T_{e 0}+T_{e 1}+T_{o 0}+T_{o 1} \leq(m+1)^{2}+4+\frac{4 n}{m}
$$

The right-hand side of (5.3) can be approximately minimized by choosing

$$
m=\left\lfloor(2 n)^{1 / 3}\right\rfloor
$$

which yields

$$
T_{e 0}+T_{e 1}+T_{o 0}+T_{o 1} \leq\left(2^{2 / 3}+2^{5 / 3}\right) n^{2 / 3}+2^{4 / 3} n^{1 / 3}+5
$$

Therefore, one of the trees must have a total length of at most one-fourth the right-hand side of (5.5), which is

$$
3 \cdot 4^{-2 / 3} n^{2 / 3}+2^{-2 / 3} n^{1 / 3}+5 / 4
$$

This gives a bound of $1 \leq \beta_{3} \leq 3 \cdot 4^{-2 / 3}<1.191$. 
In the next section, we recursively use Chung and Graham $d$-Strips to construct and analyze our heuristic in general dimension $d$.

\section{An Exponential Set of Heuristic Trees}

The goal of the next two sections is to establish an upper bound on the length of any MRST in the $d$-cube, where $d \geq 1$. We shall obtain our bound by recursively constructing $2^{d-1}$ heuristic rectilinear Steiner trees simultaneously in the cube. Each tree will have a distinct color, and we let $c(d)=2^{d-1}$ be the number of colors of our construction in dimension $d$. In this section, we outline the construction of the trees using simple generalizations of the four-color (four-tree) construction of Section 5 for $d=3$.

The basis of the recursive scheme is dimension one. Here, the $d$-cube is simply the unit interval, which we call a Chung and Graham 1-Strip. Clearly, an MRST of any set of $n$ points in the unit interval can be formed by labeling the points $x_{i}$, where $x_{1}<x_{2}<\cdots<x_{n}$, and then connecting $x_{i}$ to $x_{i+1}$ by a line segment, where $1 \leq i \leq n-1$. The length of this single MRST is at most one, so $\beta_{1}=1$ with $c(1)=1$.

For the remaining dimensions $d \geq 2$, we use a set of line segments of $[0,1]^{d}$ called a Chung and Graham d-Strip to form $c(d)$ rectilinear Steiner trees; each tree will be composed of a distinct portion of the $d$-Strip and its own set of $n$ juttings, which are wires connecting a set of $n$ points to the $d$-Strip.

To construct recursively a Chung and Graham $d$-Strip for $d \geq 2$, label any axis the $z$-axis and divide the unit interval of the axis into $m+1 z$-levels, where the levels are equally spaced and labeled as $z_{i}=i / m$, where $0 \leq \mathrm{i} \leq m$ and $m$ is fixed. In the hyperplane at each level, place a Chung and Graham $(d-1)$-Strip; then, connect the $m+1(d-1)$-Strips with $2^{d-1}$ unit line segments parallel to the $z$-axis and passing through the points $\left(z=z_{j}, \delta_{1}, \delta_{2}, \ldots, \delta_{d-1}\right)$, where $\delta_{i}=0,1$ for $1 \leq i \leq d-1$. (These points are the corners of the $(d-1)$-cube at any fixed level $z_{j}$, where $0 \leq j \leq m$.) Figure 1 shows a Chung and Graham 3-Strip, where $m=3$. Each of the four levels of the figure contains a Chung and Graham 2-Strip.

We now assign a color to each segment of the $d$-Strip. For all $0 \leq i \leq m$, label the level $z_{i}$ to be an even level if $i$ is even and an odd level if $i$ is odd. Since the hyperplane at each level contains a Chung and Graham $(d-1)$-Strip, the line segments of any level are colored using the $c(d-1)$ colors $k_{1}, k_{2}, \ldots, k_{c(d-1)}$. In the $(d-1)$-Strip at each odd level $z_{i}$, we assign a new color $k_{j}^{\prime}$ to any line segment of color $k_{j}$, where $1 \leq j \leq c(d-1)$. Hence, the $(d-1)$-Strips at the even levels remain colored with the colors $k_{j}$ and the odd levels become colored with $k_{j}^{\prime}$. To complete the coloring assignments of the $d$-Strip, arbitrarily assign a distinct color to each of the $2^{d-1}$ segments that parallel the $z$-axis; this serves to connect the $z$-levels for each color. Figure 1 illustrates the color scheme in the Chung and Graham 3-Strip, where the $c(3)=4$ colors are denoted by solid, dotted, dashed, and doubled line segments.

Given $V$, a set of $n$ points in $[0,1]^{\mathrm{d}}$, we must now connect the members of $V$ to each color of the $d$-Strip using juttings. This will be recursively accomplished 
in a manner that is analogous to the method used to connect the points to the 3-Strip in Section 5. Let $J_{d}$ be the set of juttings used to connect all the colors of the Chung and Graham $d$-Strip to $V$. In dimension one, the basis case, there is no need for juttings since all of $V$ lies in the unit interval; it is therefore convenient to define $J_{1}$ to be empty for any $V \subset[0,1]$.

For $d \geq 2$, note first that each point of $V$ belongs to one of $m^{d-1}$ naturally formed $d$-dimensional rectangular parallelepipeds, each of size 1 by $1 / m$ by $1 / m \ldots$ by $1 / m$. Henceforth, we will refer to such a rectangular parallelepiped as a $d$-box. Figure 3 depicts the nine $d$-boxes of the 3-Strip in Figure 1, and Figure 2 has isolated one of these $d$-boxes.

Consider a point $P \in V$, and let $R$ be the $d$-box containing $P$. Label the axis parallel to the sides of $R$ that have unit length the $x$-axis. Furthermore, label the two $(d-1)$-dimensional hyperplanes of the surface of $R$ that belong to adjacent $z$-levels as the top level and the bottom level. We have the following lemma.

Lemma 6.1. Each line of $R$ parallel to the $x$-axis is colored with a distinct color $k=k_{j}$ or $k=k_{j}^{\prime}$ where $1 \leq j \leq c(d-1)$ and $d \geq 2$.

Proof. We proceed by induction on $d$. For $d=2, R$ is simply a 1 by $1 / m d$-box, and by the construction of the 2-Strip, adjacent levels are distinctly colored as $k_{1}$ and $k_{1}^{\prime}$.

In dimension $d, R$ contains $2^{d-1}$ sides parallel to the $x$-axis. Half of these are in the top level of $R$, and the other half are in the bottom level. By induction, each of the top and bottom levels contains $2^{d-2}=c(d-1)$ distinctly-colored sides. But, by the construction and coloring of the $d$-Strip, there is no color in the bottom level that is the same as a color in the top level. This gives a total of $2^{d-1}=$ $2 c(d-1)$ distinct colors.

Lemma 6.1 allows us to recursively reach $P$ via juttings when $d \geq 2$. We begin by projecting $P$ to the top and bottom levels to obtain the points $P_{\mathrm{t}}$ and $P_{\mathrm{b}}$, respectively. Consider now each (unit-length) side of $R$ that parallels the $x$-axis in the top level. In the $(d-1)$-dimensional hyperplane at the top level, we recursively connect each of these sides to $P_{t}$, using the jutting scheme of dimension $d-1$. Next, we reach our destination $P$ from $P_{t}$ by directly traveling in a direction parallel to the $z$-axis.

In a manner similar to the juttings emanating from the top level, the sides of the bottom level that parallel the $x$-axis reach $P$ by first traveling recursively to $P_{\mathrm{b}}$, then directly to $P$ in a direction paralleling the $z$-axis. Lemma 6.1 guarantees that each color of the Chung and Graham $d$-Strip can reach $P$ in this way. Figure 2 illustrates a set of juttings that connect a point to the 3-Strip.

Note that in $d=2$ both $P_{\mathrm{t}}$ and $P_{\mathrm{b}}$ already lie on the 2-Strip, and the hyperplanes at the top and bottom levels of a 2-box are simply unit segments of different colors. The recursive jutting from the line segment of the top level to the point $P_{t}$ is still well defined, though, for the jutting set $J_{1}$ is empty. In addition, some point sets may exhibit a point or points that lie on the boundary of the $d$-box. In this case, some of the juttings used to reach such a boundary point may become unnecessary; 
we can consider them to have length zero. For later reference, note also that for all $d \geq 2$, every color appearing in the top (bottom) level of $R$ takes the same path paralleling the $z$-axis, from $P_{1}\left(P_{\mathrm{b}}\right)$ to $P$.

We have now constructed $c(d)$ heuristic rectilinear Steiner trees, each consisting of segments of the $d$-Strip of some color and $n$ juttings, having the same color. The next section relies on the recursive construction of the trees to calculate their combined total length.

\section{Upper Bounds in General Dimension}

This section begins with some simple lemmas that will allow us to calculate easily the combined total length of the $c(d)$ heuristic rectilinear Steiner trees constructed in Section 6, leading to an upper bound on $\beta_{d}$. We first determine the total length of the line segments of the Chung and Graham $d$-Strip.

Lemma 7.1. If $C_{d}$ is a Chung and Graham $d$-Strip, where $d \geq 1$, then

$$
\sum_{w \in C_{d}} d_{\mathrm{R}}(w)=\frac{(m+1)^{d}-2^{d}}{m-1} .
$$

Proof. Let $l_{d}=\sum_{w \in c_{d}} d_{\mathbf{R}}(w)$. For any $d \geq 2$, the $d$-Strip consists of $m+1$ Chung and Graham $(d-1)$-Strips and $2^{d-1}$ "vertical" unit line segments that parallel the $z$-axis and connect the $(d-1)$-Strips. This gives us the difference equation

$$
l_{d}=(m+1) l_{d-1}+2^{d-1} \quad \text { where } \quad d \geq 2,
$$

with

$$
l_{1}=1
$$

as a boundary condition. The right-hand side of $(7.1)$ is the solution to (7.2) and (7.3).

The next lemma computes the maximum possible jutting cost of connecting the segments of the $c(d)$ colors in a $d$-Strip to $P$, a member of a set $n$ points in the $d$-cube.

Lemma 7.2. If $J_{d}(P)$ is the set of juttings connecting the segments of the $c(d)$ colors of $a$ Chung and Graham d-Strip to $P \in[0,1]^{d}$, where $d \geq 1$, then

$$
\sum_{j \in J_{d}(P)} d_{\mathrm{R}}(j) \leq \frac{2^{d-2}(d-1)}{m} \quad \text { if } d \geq 1
$$

Proof. First, define $j_{d}^{*}=\max _{P \in[0,1]^{d}} J_{d}(P)$, where $d \geq 1$; in other words, $j_{d}^{*}$ is the greatest possible cost of connecting a point in the cube to all $c(d)$ colors of the 
$d$-Strip using juttings. In addition, let $P^{*} \in[0,1]^{d}$ be a point that attains $\sum_{j \in J_{d}\left(P^{*}\right)} d_{\mathrm{R}}(j)=j_{d}^{*}$. We will bound $\sum_{j \in J_{d}(P)} d_{\mathrm{R}}(j)$ by bounding $\sum_{j \in J_{d}\left(P^{*}\right)} d_{\mathrm{R}}(j)$.

Clearly, no juttings are needed in dimension one. For $d \geq 2$, let $R$ be the $d$-box to which $P^{*}$ belongs. By Lemma 6.1, there are $c(d-1)$ colors in both the top and bottom levels of $R$. Denote the colors of the top level by $k_{i}$, and those of the bottom level by $k_{i}^{\prime}$, where $1 \leq i \leq c(d-1)$. Now, the cost of the juttings from the $k_{i}$ to $P_{\mathrm{t}}^{*}$, the projection of $P^{*}$ onto the top level of $R$, is exactly $\sum_{j \in J_{d-1}\left(P_{*}^{*}\right)} d_{\mathrm{R}}(j)$, and the cost of connecting the $k_{i}^{r}$ of the $d$-Strip to $P_{\mathrm{b}}^{*}$, the projection of $P^{*}$ to the bottom level of $R$, is $\sum_{j \in J_{d-1}\left(P_{*}^{*}\right)} d_{\mathrm{R}}(j)$. For each pair of colors $k_{i}$ and $k_{i}^{\prime}$, the cost of reaching $P^{*}$ from $P_{\mathrm{t}}^{*}$ and $P_{\mathrm{b}}^{*}$, respectively, is $1 / m$. This gives us the recursion

$$
\begin{aligned}
j_{d}^{*} & =\sum_{j \in J_{d-1}\left(P_{*}^{*}\right)} d_{\mathrm{R}}(j)+\sum_{j \in J_{d-1}\left(P_{\mathrm{b}}^{*}\right)} d_{\mathrm{R}}(j)+\frac{c(d-1)}{m} \\
& \leq 2 j_{d-1}^{*}+\frac{2^{d-2}}{m}, \quad \text { where } \quad d \geq 2,
\end{aligned}
$$

with boundary condition

$$
j_{1}=0
$$

This implies

$$
\frac{j_{d}^{*}}{2^{d-1}}-\frac{j_{d}^{*}-1}{2^{d-2}} \leq \frac{1}{2 m}
$$

and

$$
\frac{j_{d}^{*}}{2^{d-1}}-j_{1} \leq \sum_{i=2}^{d} \frac{1}{2 m}
$$

so $j_{d}^{*} \leq 2^{d-2}(d-1) m^{-1}$. By the definition of $j_{d}^{*}$, the proof is complete since $\sum_{j \in J_{d}(P)} d_{\mathrm{R}}(j) \leq j *$

We have now assembled all the components necessary to bound the $\beta_{d}$. The Chung and Graham $d$-Strip combined with the jutting set $J_{d}=\bigcup_{P \in V} J_{d}(P)$ gives us $c(d)$ trees, having total length

$$
\sum_{w \in C_{d}} d_{\mathrm{R}}(w)+\sum_{w \in J_{d}} d_{\mathrm{R}}(w) \leq \frac{(m+1)^{d}-2^{d}}{m-1}+\frac{2^{d-2}(d-1) n}{m},
$$

where $d \geq 1$ and we have applied Lemmas 7.1 and 7.2. To approximately minimize the right-hand side of (7.9), choose

$$
m=\left\lfloor\left(2^{d-2} n\right)^{1 / d}\right\rfloor
$$


to produce

$$
\sum_{w \in C_{d}} d_{\mathrm{R}}(w)+\sum_{w \in J_{d}} d_{\mathrm{R}}(w) \leq d 2^{(d-1)(d-2) / d} n^{(d-1) / d}+o\left(n^{(d-1) / d}\right)
$$

Since there are $c(d)$ trees, there must exist some rectilinear Steiner tree $T$ such that

$$
\sum_{w \in T} d_{\mathbf{R}}(w) \leq \frac{\sum_{w \in C_{d}} d_{\mathbf{R}}(w)+\sum_{w \in J_{d}} d_{\mathbf{R}}(w)}{2^{d-1}} \leq d\left(\frac{n}{4}\right)^{(d-1) / d}+o\left(n^{(d-1) / d}\right)
$$

which yields the bound of Theorem 2 .

\section{Conclusion}

The heuristic used to prove bounds on the $\beta_{a}$ is one of many possible "strip" methods available for developing upper bounds on objects like the MRST in $[0,1]^{d}$. Many such heuristics have been used to attack the traveling salesman problem. For example, Goldstein and Reingold (1987) seek to minimize distance due to juttings by forming an exponential number of tours, each occupying a translated version of the cube. Goddyn (1990) uses optimal quantizers to select a particular strip that undergoes an infinite number of translations, building an infinite number of tours. But, for the MRST, a method similar to that of Goldstein and Reingold (1987) and a method using an infinite number of translations similar to Goddyn (1990) each produce the same results as the generalized Chung and Graham method used here to produce Theorem 2.

Karloff (1989) and Moran (1984) choose the midpoints of two points that are relatively close together and build traveling salesman tours that pass through the midpoints, then obtain bounds by modifying these tours to include the original pairs of points. Unfortunately, the rectilinear metric does not benefit from these midpoint methods, which are well-suited for the Euclidean metric. It seems, then, that the bounds of Theorem 2 may be the best possible bounds resulting from a strip heuristic, and that a new bag of tricks will be required to improve them. Perhaps Smith's methods (Smith (1988)) could be refined for this use.

A determination of the exact values of $\beta_{d}$ for $d \geq 3$ is an open problem. Chung and Graham's $\beta_{2}=1$ is one of the only known nontrivial constants in the area of worst-case weights for problems of combinatorial optimization. Supowit et al. (1983) have conjectured that if $\beta_{\mathrm{TSP}, \mathrm{d}}$ and $\beta_{\mathrm{MST}, d}$ are the worst-case constants associated with the traveling salesman and minimum spanning tree problems, respectively, then $\beta_{\mathrm{TSP}, d}=\beta_{\mathrm{MST}, d}$. We append this to conjecture that

$$
\beta_{\mathrm{TSP}, d}=\beta_{\mathrm{MST}, d}=\beta_{d} .
$$

Chung and Graham (1981) modify their construction in $d=2$ to lessen the constants associated with lower order terms; though our method leaves room for 
similar modifications, we have made no attempt to do so here. In addition, Chung and Graham conjecture that in $d=2$, for all $n \geq 1, s^{*}(n) \leq \sqrt{n}+1$; this conjecture remains open.

Also open is the asymptotic behavior of the Euclidean minimum Steiner tree. A theorem like Theorem 1 for the Euclidean case could be a by-product of our proof here, but the existence of Steiner points in the intercellular spaces of the construction in Section 2 is problematic. Here we used the Generalized Hanan Theorem of Snyder (1992) to deal with such points, but, unfortunately, there is no analogous theorem for the Euclidean case.

A final open problem deals with the length of the worst-case MRST as the dimension $d$ varies. Goddyn (1990) considers for the Euclidean traveling salesman problem the existence of $\lim _{d \rightarrow \infty} \beta_{\mathrm{TSP}, d} \sqrt{d}$. We can ask similar questions for the MRST problem; but, since the previous work on this problem assumes that $d$ is fixed, to investigate such questions will most likely require new methodologies.

\section{References}

Beardwood, J., Halton, J. H., and Hammersley, J. M. (1959). The shortest path through many points, Math. Proc. Cambridge Philos. Soc. 55, 299-327.

Bern, M. W. (1988). Two probabilistic results on rectilinear Steiner trees, Algorithmica 3, 191-204.

Chung, F. R. K. and Graham, R. L. (1981). On Steiner trees for bounded point sets, Geom. Dedicata 11, 353-361.

Chung, F. R. K. and Hwang, F. K. (1979), The largest minimal rectilinear Steiner trees for a set of $n$ points enclosed in a rectangle with given perimeter, Networks 9, 19-36.

Courant, R. and Robbins, H. (1941). What is Mathematics?, Oxford University Press, New York.

Few, L. (1955). The shortest path and the longest road through $n$ points in a region, Mathematika 2 , $141-144$.

Foulds, L. R., Hendy, M. D., and Penny, D. (1979). A graph-theoretic approach to the development of minimal phylogenetic trees, J. Mol. Evol. 13, 127-149.

Garey, M. R., Graham, R. L., and Johnson, D. S. (1976). Some NP-complete geometric problems, Proceedings of the Eighth Annual ACM Sympositum on Theory of Computing, Association for Computing Mach., New York, pp. 10-22.

Garey, M. R. and Johnson, D. S. (1977). The rectilinear Steiner tree problem is NP-complete, SIAM J. Appl. Math. 32, 826-834.

Gilbert, E. N. and Pollak, H. O. (1968). Steiner minimal trees, SIAM J. Appl. Math. 16, 1-29.

Goddyn, L. (1990). Quantizers and the worst-case Euclidean traveling salesman problem, submitted to J. Combin. Theory, Ser. B. 50, 65-81.

Goldstein, A. S. and Reingold, E. M. (1987). Improved Bounds on the Traveling Salesman Problem in the Unit Cube, Technical Report, Department of Computer Science, University of Illinois, Urbana-Champaign, Illinois.

Hanan, M. (1966). On Steiner's problem with rectilinear distance, J. SIAM Appl. Math. 14, 255-265.

Hwang, F. K. (1976). On Steiner minimal trees with rectilinear distance, SIAM J. Appl. Math. 30, 104-114.

Karlof, H. J. (1989). How long can a Euclidean traveling salesman tour be? SIAM J. Discrete Math. 2, 91-99.

Melzak, Z. A. (1961). On the problem of Steiner, Canad. Math. Bull. 4, 143-148.

Moran, S. (1984). On the length of optimal TSP circuits in sets of bounded diameter, J. Combin. Theory, Ser. B 37, 113-141.

Sankoff, D. and Rousseau, P. (1975). Locating the vertices of a Steiner tree in an arbitrary metric space, Math. Programming 9, 240-246. 
Smith, W. D. (1988). Studies in Computational Geometry Motivated by Mesh Generation, Ph.D. thesis, Program in Applied and Computational Mathematics, Princeton University, Princeton, N.J.

Snyder, T. L. (1987). Asymptotic Worst-Case Lengths in Some Problems from Classical Computational Geometry and Combinatorial Optimization, Ph.D. thesis, Princeton University, Princeton, N.J.

Snyder, T. L. (1991). Lower bounds for rectilinear Steiner trees in bounded space, Inform. Process. Lett. $37,71-74$.

Snyder, T. L. (1992). On the exact location of Steiner points in general dimension, SIAM J. Comput. 21, $163-180$.

Snyder, T. L. and Steele, J. M. (1990). Worst-case greedy matchings in the unit $d$-cube, Networks 20, $779-800$.

Steele, J. M. (1981). Subadditive Euclidean functionals and non-linear growth in geometric probability, Ann. Probab. 9, 365-376.

Steele, J. M. and Snyder, T. L. (1989). Worst-case growth rates of some problems from combinatorial optimization, SIAM J. Comput. 18, 278-287.

Supowit, K. J., Reingold, E. M., and Plaisted, D. A. (1983). The traveling salesman problem and minimum matchings in the unit square, SIAM J. Comput. 12, 144-156.

Received July 16, 1990, and in revised form June 13, 1991. 